\title{
Defining a rational step-care algorithm for managing thyroid carcinoma patients with elevated thyroglobulin and negative on radioiodine scintigraphy (TENIS): considerations and challenges towards developing an appropriate roadmap
}

\author{
Sandip Basu ${ }^{1}$ - Mitali Dandekar ${ }^{2}$ - Amit Joshi ${ }^{3}$ - Anil D'Cruz ${ }^{2}$ \\ Received: 25 March 2015 / Accepted: 31 March 2015 /Published online: 20 May 2015 \\ (C) Springer-Verlag Berlin Heidelberg 2015
}

One of the most challenging clinical situations in patients with differentiated thyroid cancer (DTC) is an elevated serum thyroglobulin ( $\mathrm{Tg}$ ) level with negative radioiodine scintigraphy (commonly termed "TENIS"). The diagnostic and therapeutic approaches adopted by attending physicians in day-to-day routine vary quite widely. The main reason for this variation is the rarity of the condition $(2-13 \%$ from various series reported in literature), and its management, especially therapeutic strategies, continues to be imperfect in the absence of a clear-cut understanding and lack of evidence of its aetiology, as observed by various authorities [1]. In this editorial, we discuss the different therapeutic strategies that have been explored and the associated pitfalls in interpretation of clinical findings, and propose a logical step-wise algorithmic approach to care to follow in routine clinics in this particular group of patients. Our views are supported by an overview of the evidence in the literature (with respect to both efficacy and adverse effects) and by practical experience. In addition, we critically review and analyse the steps needed in the immediate future to enhance and rationalize patient care. We believe that

Sandip Basu

drsanb@yahoo.com

1 Radiation Medicine Centre, Bhabha Atomic Research Centre (BARC), Tata Memorial Hospital, Annexe building, Jerbai Wadia Road, Parel, Mumbai 400 012, India

2 Department of Surgical Oncology-Head and Neck Services, Tata Memorial Hospital, Mumbai, India

3 Department of Medical Oncology, Tata Memorial Hospital, Mumbai, India such endeavours to identify and prioritize the challenges will further the roadmap to the development of a systematic and scientific approach to the management of patients with TENIS.

\section{Diagnostic investigation protocol and interpretive pitfalls}

In a patient with TENIS presenting in the clinic, the usual initial investigations are (1) reliable neck ultrasonography (neck lymph nodal metastases being the commonest site of disease recurrence) and (2) whole-body FDG PET/CT. However, four factors (Fig. 1) need to be considered before TENIS can be diagnosed. First, iodide interference should be ruled out (a factor of paramount importance even in the days of substantial patient knowledge and information). Second, the imaging characteristics of ${ }^{131}$ I are relatively inferior to those of ${ }^{123} \mathrm{I}$, which could partly explain visualization of iodine-concentrating foci on posttreatment whole-body scans that were negative on diagnostic study. This is particularly relevant in developing countries where ${ }^{131}$ I continues to be commonly employed for thyroid imaging due to non-availability of ${ }^{123} \mathrm{I}$. Third, a false-positive elevated $\mathrm{Tg}$ level related to heterophile antibody and anti-Tg antibody (which of course is much less common than the false-negative test) should be excluded. Finally, nodal recurrence should be ruled out. Such nodes are frequently negative for sodium-iodide symporter and may therefore not show up on a routine scan. If all these pitfalls are ruled out with certainty, the patient is considered to have true TENIS syndrome and an attempt is made to find treatable disease foci. 


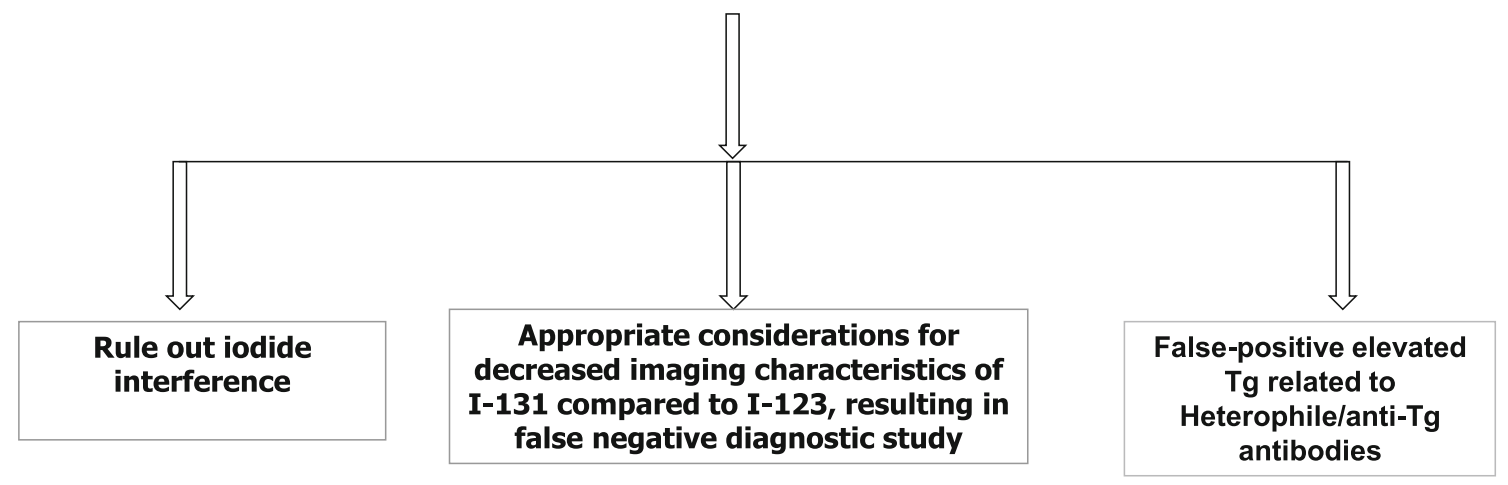

Fig. 1 Factors to consider before making a diagnosis of TENIS

\section{Routine treatment approaches: considerations for developing an algorithm for the management of TENIS}

The proposed algorithm developed for the management of TENIS is shown in Fig. 2.

Defining part of the treatment algorithm is relatively straightforward: when operable disease is detected (e.g. neck lymphadenopathy) as the sole site of metastatic disease, appropriate surgery is the treatment of choice. With respect to distant disease, operability needs to be considered on a caseby-case basis (such as in solitary flat metastases in bone or brain metastases) in terms of the ease of achieving an appropriate reconstruction. Pulmonary metastases, on the other hand are frequently multiple and in most cases, unresectable. Empirical radioiodine therapy needs special mention here. As stated above, visualization of metastatic thyroid cancer on the posttreatment scan that was invisible on the diagnostic study relates to two factors: (1) poor imaging characteristics of ${ }^{131} \mathrm{I}$ and (2) enhanced sensitivity related to dose responsive radioiodine imaging. Both factors separately or together play a role in this visualization discrepancy and the condition in a patient with these findings may not be true TENIS.

A decrease in serum Tg after empirical therapeutic doses of ${ }^{131} \mathrm{I}$ has been found and is associated with better survival, but this needs further evaluation. Ma et al. [2], in a metaanalysis of 314 patients, found that $62 \%$ of the patients showed radioiodine-positive posttreatment whole-body scanning results while $63 \%$ of 271 patients, in whom follow-up data were available, achieved a decrease in Tg level. However, it is not clear whether the decreases in Tg levels occurred primarily in patients demonstrating radioiodine-positive disease on the posttreatment scan. This is an area that needs to be researched further to define the role of ${ }^{131} \mathrm{I}$ therapy and the
Fig. 2 Decision-making stepwise algorithm for the management of TENIS with routine treatment approaches

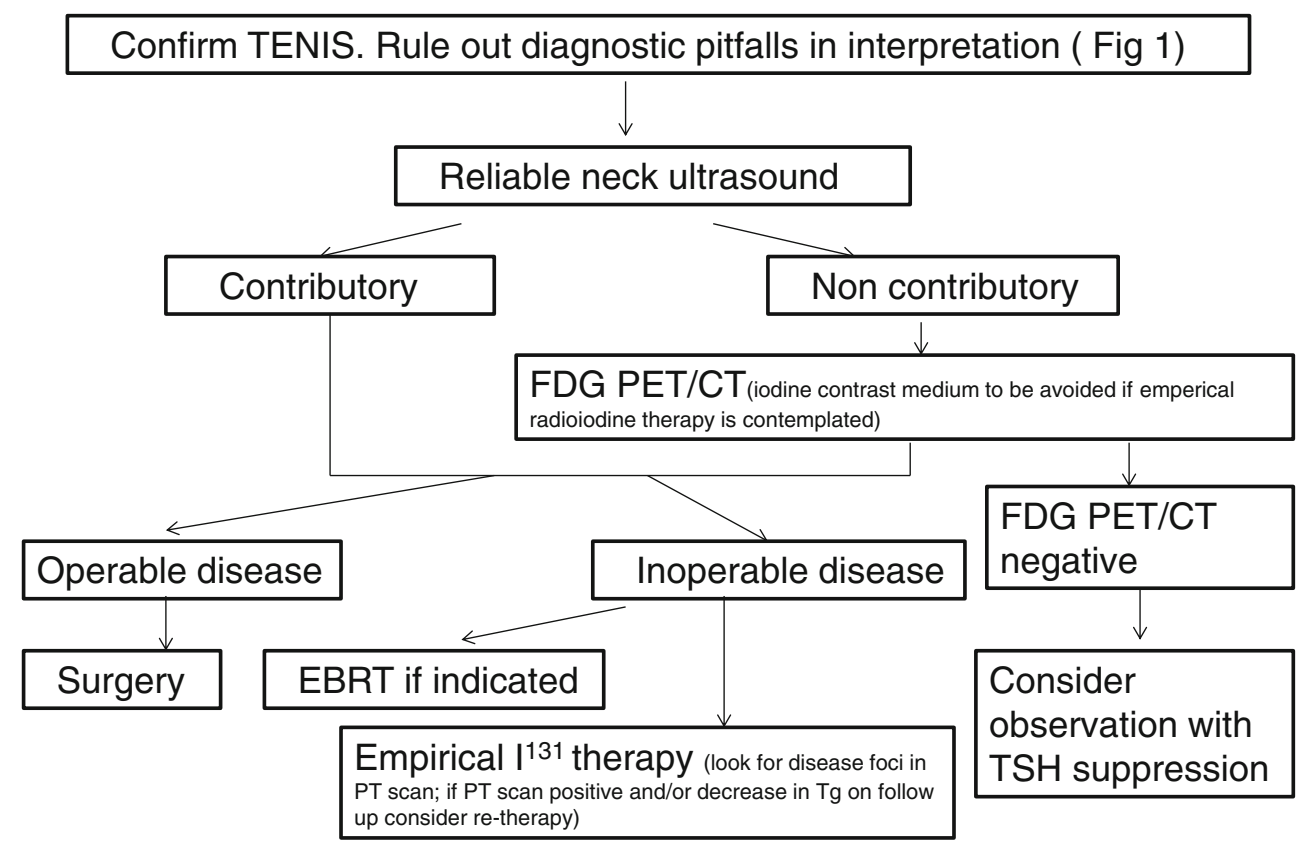


future of this treatment approach, particularly weighing its advantages against its potential toxicity. It is of course clear that such therapy needs to be considered when (1) surgery is not feasible and (2) the Tg level is high (the usual consensus is that the value should be at least more than $10 \mathrm{ng} / \mathrm{ml}$ ) or showing a fast doubling time [3]. Particular consideration should be given to the finding of "elevated Tg but normal FDG PET scan" which is a real entity found in practice in patients with DTC. A negative FDG PET scan without any obvious anatomically delineated disease in the setting of a raised Tg level is a strong favourable prognostic marker for predicting a prolonged symptom-free status in most patients and hence may not justify empirical ${ }^{131}$ I therapy [4].

In unresectable disease, external radiotherapy is an option, although its use should be properly justified because of its obvious adverse effects when used in the neck region, such as tracheitis, tracheal stenosis, oesophagitis and very prominent neck fibrosis (hence it should be employed with caution if future neck surgery is contemplated). Its use in painful bony metastases and brain metastasis (that has lost the ability to concentrate ${ }^{131} \mathrm{I}$ ) is of course indicated as it can improve the quality of life in these patients.

\section{Investigational treatment approaches: the potentials, challenges and their probable place in the management algorithm}

A scheme for the selection of therapeutic agent for the treatment of TENIS is shown in Fig. 3.

Investigational treatment approaches can be classified into four major domains: (1) redifferentiation therapy, (2) molecular targeted therapy with tyrosine kinase inhibitors (TKI) and cyclooxygenase 2 inhibitors, (3) chemotherapy, and (4) peptide receptor radionuclide therapy (PRRT). The redifferentiation agents that have been investigated in thyroid carcinoma [5-11] include: retinoic acid, resveratrol, histone deacetylase inhibitors (e.g. suberoylanilide hydroxamic acid, depsipeptide), peroxisome proliferator-activated receptor agonists (e.g. the antidiabetic agent rosiglitazone), specific inhibitors of the mTOR pathway (e.g. temsirolimus) and heat shock protein 90 inhibitor (e.g. tanespimycin). Most of these have been examined in in-vitro studies, while clinical trials and experience with retinoic acid and rosiglitazone have produced inconsistent results.

The usual consensus on actively considering systemic therapy is in patients with radioiodine-negative/resistant disease, in patients with progressive disease and in patients in whom a "wait and watch" strategy is not acceptable. The documentation of disease progression (either on FDG PET/CT or with other anatomical modalities) is important as TKIs produce a tumoristatic response as opposed to a tumoricidal effect. In some patients with radioiodine-refractory thyroid cancer and even in some FDG PET-positive patients, metastatic disease can remain stable for months. Also, as mentioned above, a negative FDG PET scan without any obvious anatomically delineated disease in the setting of raised $\mathrm{Tg}$ level usually carries a good prognosis and such patients would not be candidates for this type of therapy. Asymptomatic patients with indolent disease may not benefit from TKI therapies. This is important as side effects of TKIs may adversely affect a patient's quality of life. In contrast, patients with more rapidly progressive disease may benefit from the early use of TKIs, even if they are associated with some adverse side effects [12, 13]. TKI therapies target multiple oncoproteins and oncogenic signalling pathways. TKIs inhibit activation of the mitogenactivated protein kinase pathway, the PI3K-AKT pathway in tumour cells, and vascular endothelial growth factor receptor.
Fig. 3 Therapeutic agent selection for the treatment of TENIS

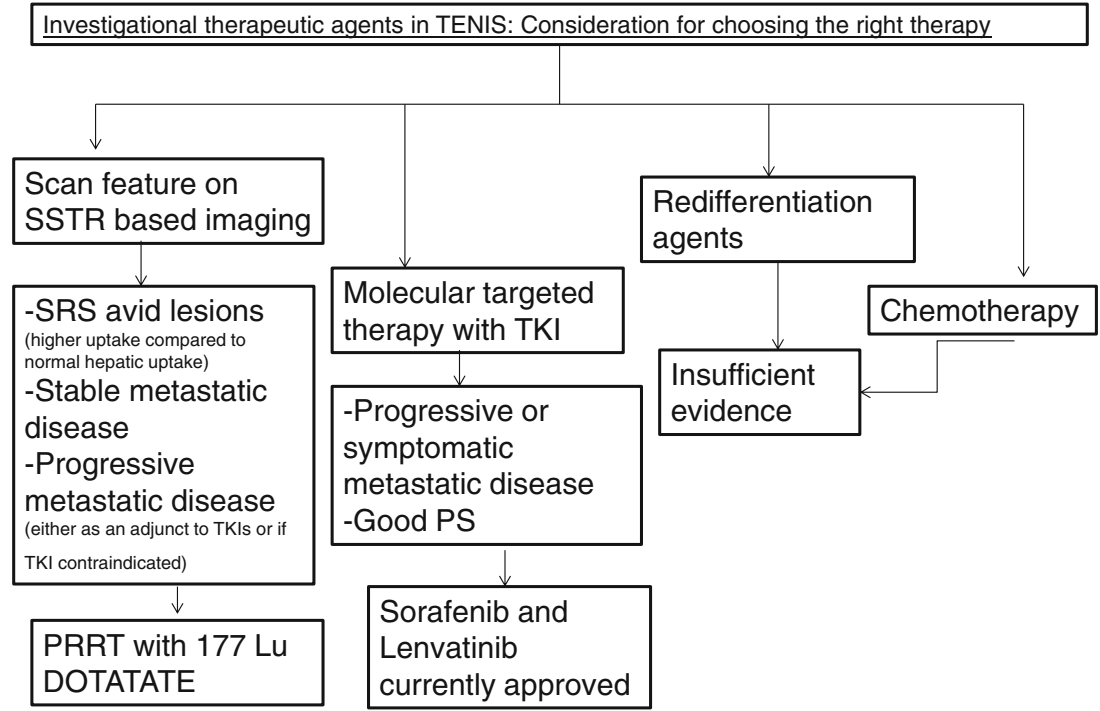


Amongst the molecular targeted therapies [13-22] with TKI, the result with sorafenib and lenvatinib are most promising and both of these are approved by the US Food and Drug Administration for use in this setting. In a phase III trial (DECISION), 417 patients with radioiodine-refractory DTC with locally advanced or metastatic disease who had radiographically progressed within 14 months of entry were randomized to either sorafenib or placebo. Median progression-free survival was improved from 5.8 months on placebo to 10.8 with sorafenib [21]. This was associated with significant toxicities leading to dose modification in $78 \%$ of patients and permanent discontinuation of therapy in $19 \%$ of patients. Another TKI, lenvatinib, was used in a phase III randomized double-blind trial in 392 radioiodine-refractory patients. The median progression-free survival was 18.3 versus 3.6 months in favour of lenvatinib as compared with placebo. Similarly, the rate of discontinuation of lenvatinib due to adverse effects was $14.2 \%$ [23]. Although most responses with these agents have been reported to produce "stable disease", in practice their use is associated with substantial comorbidities leading to drug discontinuation. Toxicities of these TKIs include hand-foot skin reaction, risk of cardiac ischaemia and/or infarction, haemorrhage, hypertension, risk of QT interval prolongation and drug-Induced hepatitis. The authors' own experience with these agents is that the adverse symptoms are a major issue, particularly when dealing with a patient with aggressive disease and relatively poor general condition.

Other agents under investigation in various phase 2 studies are vemurafenib (a selective inhibitor of the V600E mutant BRAF kinase), selumetinib which selectively inhibits MEK 1 and MEK 2, sunitinib and pazopanib. In addition to the toxicity of these new drugs, their cost is also an important consideration. Most patients need to remain on these agents until progression. Chemotherapy with standard agents (e.g. doxorubicin, cisplatin and taxanes) and thalidomide has not been popular because of unconvincing results with significant toxicity, whereas a newer thalidomide analogue lenalidomide needs to be investigated further [23-28]. To summarize, the major issues with these agents have been the requirement for more consistent results from multiple centres that would further validate the initially reported promising results. Unacceptable toxicity is another reason for unpopularity amongst patients who are otherwise symptom-free with thyroxine suppression alone.

In recent years, PRRT with ${ }^{177} \mathrm{Lu} /{ }^{90} \mathrm{Y}$-DOTATATE has been increasingly tested [29-31] in this group of patients because some demonstrate increased tracer uptake on somatostatin receptor (SSTR) imaging. However, the grade of uptake needs to be determined and patients with high uptake (compared to the normal hepatic uptake) are candidates for this form of therapy. The results are preliminary and therefore cannot be commented upon, but our initial experience is that, although $63 \%$ of patients show SSTRpositive lesions on imaging, $15 \%$ qualify for PRRT according to the strict criterion for patient selection for PRRT. However, unlike gastroenteropancratic tumours, SSTR-expressing DTC shows an inferior response. An area of research that could be to explored is the SSTR profile in DTC in contrast to the profiles in GEP and MTC.

\section{Can we individualize or risk-stratify the experimental approaches in TENIS?}

Based on the above discussion, we propose an individualization scheme for adopting experimental approaches in patients with TENIS with inoperable disease. To evolve a rational strategy, we may divide patients into three groups based upon the biology and aggressiveness of their disease:

1. In patients with FDG PET/CT-negative scans or with no other anatomically detected disease, levothyroxine suppression and surveillance should suffice.

2. In patients with stable disease detected anatomically or on FDG PET/CT, SSTR-targeted ${ }^{68}$ Ga-DOTANOC/TATE $\mathrm{PET} / \mathrm{CT}$ imaging may be considered to explore the possibility of PRRT. If the metastatic lesions show high-grade uptake, PRRT could be considered as the first option.

3. In patients with fast-growing disease (a definition that is yet to be determined), TKI with or without PRRT (if found to be suitable on diagnostic imaging) could be the preferred plausible approach. PRRT is feasible in this group only if there are SRS-avid metastatic lesions with higher uptake than normal hepatic uptake. PRRT could be used in two settings in this subgroup: (1) as an adjunct to TKIs, and (2) if TKIs are contraindicated due associated morbidities.

\section{Conclusion: need for prioritising the challenging issues for an appropriate roadmap}

The management of TENIS is in evolution. Parallel to the search for promising therapeutic agents, identification of the priority areas for research is equally important. The commonly perceived needs at present appear to be: (1) estimating definitive rates of response to PRRT and TKIs in various subgroups of patients with TENIS through multicentre prospective studies, (2) establishing the role of TKIs and PRRT in TENIS in patients with stable DTC versus patients with progressive DTC, (3) coupled with the first two needs, performing more rigorous basic science research to find an appropriate redifferentiation agent, and (4) determining SSTR expression profiles in TENIS with varying disease biology. This multipronged approach is likely to result in further progress towards the scientific management of this group of patients. 
Conflicts of interest None.

\section{References}

1. Silberstein EB. The problem of the patient with thyroglobulin elevation but negative iodine scintigraphy: the TENIS syndrome. Semin Nucl Med. 2011;41(2):113-20.

2. Ma C, Xie J, Kuang A. Is empiric 131I therapy justified for patients with positive thyroglobulin and negative 131I whole-body scanning results? J Nucl Med. 2005;46(7):1164-70.

3. Mazzaferri EL. Empirically treating high serum thyroglobulin levels. J Nucl Med. 2005;46:1079-86.

4. Vural GU, Akkas BE, Ercakmak N, Basu S, Alavi A. Prognostic significance of FDG PET/CT on the follow-up of patients of differentiated thyroid carcinoma with negative 131I whole-body scan and elevated thyroglobulin levels: correlation with clinical and histopathologic characteristics and long-term follow-up data. Clin Nucl Med. 2012;37(10):953-9.

5. Grunwald F, Menzel C, Bender H, Palmedo H, Otte R, Fimmers R, et al. Redifferentiation therapy induced radioiodine uptake in thyroid cancer. J Nucl Med. 1998;39:1903-6.

6. Furuya F, Shimura H, Suzuki H, Taki K, Ohta K, Haraguchi K, et al. Histone deacetylase inhibitors restore radioiodide uptake and retention in poorly differentiated and anaplastic thyroid cancer cells by enhancing expression of the sodium/iodide symporter, thyroperoxidase and thyroglobulin. Endocrinologist. 2004;145: 2865-75.

7. Shen WT, Chung WY. Treatment of thyroid cancer with histone deacetylase inhibitors and peroxisome proliferator-activated receptor-gamma agonists. Thyroid. 2005;15:594-9.

8. Luong QT, O'Kelly J, Braunstein GD, Hershman JM, Koeffler HP. Antitumor activity of suberoylanilide hydroxamic acid against thyroid cancer cell lines in vitro and in vivo. Clin Cancer Res. 2006;12: 5570-7.

9. Liu D, Xing J, Trink B, Xing M. BRAF mutation-selective inhibition of thyroid cancer cells by the novel MEK inhibitor RDEA119 and genetic-potentiated synergism with the mTOR inhibitor temsirolimus. Int J Cancer. 2010;127(12):2965-73.

10. Sebai H, Hovsepian S, Ristorcelli E, Aouani E, Lombardo D, Fayet G. Resveratrol increases iodine trapping in the rat thyroid cell line FRTL-5. Thyroid. 2010;20:195-203.

11. Morris JC. Resveratrol, thyroid cancer and iodide: drink up? Thyroid. 2010;20:125-6.

12. National Comprehensive Cancer Network. Guidelines for the treatment of thyroid carcinoma. 2014 http://www.nccn.org/ professionals/physician_gls/pdf/thyroid.pdf.

13. Cabanillas ME, Waguespack SG, Bronstein Y, Williams MD, Feng L, Hernandez M, et al. Treatment with tyrosine kinase inhibitors for patients with differentiated thyroid cancer: the M. D. Anderson experience. J Clin Endocrinol Metab. 2010;95(6):2588-95.

14. Gupta-Abramson V, Troxel AB, Nellore A, Puttaswamy K, Redlinger M, Ransone K, et al. Phase II trial of sorafenib in advanced thyroid cancer. J Clin Oncol. 2008;26:4714-9.

15. Cohen EEW, Rosen LS, Vokes EE, Kies MS, Forastiere AA, Worden FP, et al. Axitinib is an active treatment for all histologic subtypes of advanced thyroid cancer: results from a phase II study. J Clin Oncol. 2008;26:4708-13.

16. Sherman SI, Wirth LJ, Droz J-P. Motesanib diphosphate in progressive differentiated thyroid cancer. N Engl J Med. 2008;359:31-42.

17. Pennell NA, Daniels GH, Haddad RI. A phase II study of gefitinib in patients with advanced thyroid cancer. Thyroid. 2008;3:317-23.

18. Dawson SJ, Conus NM, Toner GC, Raleigh JM, Hicks RJ, McArthur G, et al. Sustained clinical responses to tyrosine kinase inhibitor sunitinib in thyroid carcinoma. Anticancer Drugs. 2008;19:547-52.

19. Kloos RT, Ringel MD, Knopp MV, Hall NC, King M, Stevens R, et al. Phase II trial of sorafenib in metastatic thyroid cancer. J Clin Oncol. 2009;27:1675-84.

20. Mrozek E, Kloos RT, Ringel MD, Kresty L, Snider P, Arbogast D, et al. Phase II study of celecoxib in metastatic differentiated thyroid carcinoma. J Clin Endocrinol Metab. 2006;91:2201-4.

21. Brose MS, Nutting CM, Jarzab B, Elisei R, Siena S, Bastholt L, et al. Sorafenib in radioactive iodine-refractory, locally advanced or metastatic differentiated thyroid cancer: a randomised, double-blind, phase 3 trial. DECISION investigators. Lancet. 2014;384(9940):319-28.

22. Schlumberger M, Tahara M, Wirth LJ, Robinson B, Brose MS, Elisei R, et al. Lenvatinib versus placebo in radioiodine-refractory thyroid cancer. N Engl J Med. 2015;372(7):621.

23. Droz JP, Schlumberger M, Rougier P, Ghosn M, Gardet P, Parmentier C. Chemotherapy in metastatic nonanaplastic thyroid cancer: experience at the Institut Gustave-Roussy. Tumori. 1990;76:480-3

24. Santini F, Bottici V, Elisei R, Montanelli L, Mazzeo S, Basolo F, et al. Cytotoxic effects of carboplatinum and epirubicin in the setting of an elevated serum thyrotropin for advanced poorly differentiated thyroid cancer. J Clin Endocrinol Metab. 2002;87:4160-5.

25. Ain KB, Lee C, Williams KD. Phase II trial of thalidomide for therapy of radioiodine unresponsive and rapidly progressive thyroid carcinomas. Thyroid. 2007;17:663-70.

26. Ain KB, Lee C, Holbrook KM, Dziba JM, Williams KD. Phase II study of lenalidomide in distantly metastatic, rapidly progressive, and radioiodine-unresponsive thyroid carcinomas: preliminary results. J Clin Oncol. 2008;26(15S):6027. Abstract.

27. Kotla V, Goel S, Nischal S, Heuck C, Vivek K, Das B, et al. Mechanism of action of lenalidomide in hematological malignancies. Hematol Oncol. 2009;2:36-8.

28. Sherman SI. Cytotoxic chemotherapy for differentiated thyroid carcinoma. Clin Oncol. 2010;22:464-8.

29. Teunissen JJ, Kwekkeboom DJ, Krenning EP. Staging and treatment of differentiated thyroid carcinoma with radiolabeled somatostatin analogs. Trends Endocrinol Metab. 2006;17:19-25.

30. Jois B, Asopa R, Basu S. Somatostatin receptor imaging in non(131)I-avid metastatic differentiated thyroid carcinoma for determining the feasibility of peptide receptor radionuclide therapy with (177)Lu-DOTATATE: low fraction of patients suitable for peptide receptor radionuclide therapy and evidence of chromogranin A level-positive neuroendocrine differentiation. Clin Nucl Med. 2014;39(6):505-10.

31. Iten F, Muller B, Schindler C, Rasch H, Rochlitz C, Oertli D, et al. [(90)Yttrium-DOTA]-TOC response is associated with survival benefit in iodine-refractory thyroid cancer: long-term results of a phase 2 clinical trial. Cancer. 2009;1154:2052-62. 